\title{
SOLUTION VERIFICATION STUDIES OF A PRESSURE-BASED COMPRESSIBLE FLOW SOLVER
}

\author{
João Muralha ${ }^{1}$, Cristiano Silva ${ }^{1}$, Luís Eça ${ }^{1}$ and Christiaan M. Klaij ${ }^{2}$ \\ ${ }^{1}$ Instituto Superior Técnico - Universidade de Lisboa, Av. Rovisco Pais 1, 1049-001, Lisbon, Portugal, \\ \{joao.muralha, cristiano.silva, luis.eca\}@tecnico.ulisboa.pt \\ ${ }^{2}$ Maritime Research Institute Netherlands, P.O.Box 28, 6700AA Wageningen, The Netherlands, \\ c.klaij@marin.nl
}

Key words: Compressible Flow, Numerical Errors, Iterative and Grid Convergence

\begin{abstract}
Although considering the fluid to be incompressible is a common and valid approximation in most hydrodynamic simulations, certain phenomena like sloshing or slamming involve compressibility effects. In order to capture such effects, the maritime CFD code ReFRESCO is being extended with a compressible flow solver for the air in two-phase flow simulations. The compressible Navier-Stokes equations, discretized with a cell-centered, collocated finite volume method, are solved with a pressurebased SIMPLE algorithm that is compatible with the incompressible flow solver and enforces pressurevelocity-density coupling with a pressure-correction equation and an equation of state. In this paper, the compressible solver is tested for subsonic, transonic and supersonic flow of an inviscid perfect gas in a channel with an arc circular bump. We confirm that the pressure-based solver can indeed achieve iterative convergence to levels close to machine accuracy for all three regimes, with moderate decrease of convergence rate at higher Mach numbers and on finer grids. Grid refinement studies are performed to determine its accuracy and show observed orders of grid convergence between one and two for different quantities and different convection schemes, with lowest order for the supersonic regime, as expected. Finally, we notice that monotonic grid convergence can be attained if the grids are refined far beyond the levels typically reported in open literature.
\end{abstract}

\section{INTRODUCTION}

In many Maritime applications, it is assumed incompressible flow, i.e., for each material particle in the flow density remains constant. Although reasonable and accurate for the majority of the computational simulations performed in the Offshore and Naval industry, some phenomena require different modelling approximations. Sloshing, and slamming are examples of phenomena, where compressibility effects must be considered. In order to capture such effects, the maritime CFD code ReFRESCO [1] is being extended with a compressible flow solver for the air in two-phase flow simulations. This is done in two steps: first, a single-phase solver of compressible perfect gas flow is coded and tested, and then this solver is extended to deal with multi-phase (in)compressible flows. The present study aims to check the numerical robustness of the single-phase solver by performing a solution verification study.

Following the definition of verification and validation given by Roache [2]. Verification is a purely mathematical exercise that intends to guatantee that the equations are solved correctly, while validation 
is used to check if the chosen mathematical model is suitable to tackle a selected physical problem, i.e., it answers the question: are the right equations being solved? Furthermore, verification is divided in two subcategories: code verification and solution verification. The purpose of code verification is to make sure that there are no coding mistakes, while the goal of solution verification is to estimate the numerical errors. As stated, the focus of this work is in solution verification, because code verification of the solver was previously performed in [3]

Navier-Stokes solvers can be classified as pressure-based or density-based. In a density-based solver, density is calculated from the continuity equation, and pressure is obtained from an equation of state. For pressure-based methods, a pressure equation is obtained from the continuity and momentum equations, coupling velocity and pressure, and enforcing mass conservation. Historically pressure-based solvers were developed to deal with the pressure-velocity decoupling in incompressible flows, while densitybased solvers were used in the aerospace industry to simulate transonic and supersonic flows. Since they were introduced pressure-based $[4,5]$ and density-based $[6,7]$ methods have been modified to deal with flows outside their original speed range. Taking this into consideration, the readily available ReFRESCO incompressible pressure-based method was considered a good starting point for the implementation of a pressure-based compressible flow solver.

The numerical convergence properties of the solver, i.e., the robustness (iterative errors) and the order of grid convergence (discretization error), were previously assessed for turbulent flows at low Mach number. So in the present work the focus is shifted to the simulation of high Mach number flows including shocks. To this end we have selected the subsonic, transonic and supersonic flows over an arc bump. Since it was

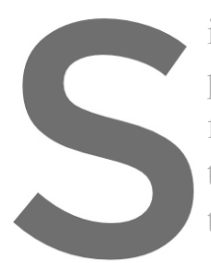
introduced in the GAMM confere pressure-based $[4,5]$ and density flow throughout a channel with a the thickness to chord ratic the supersonic test case.
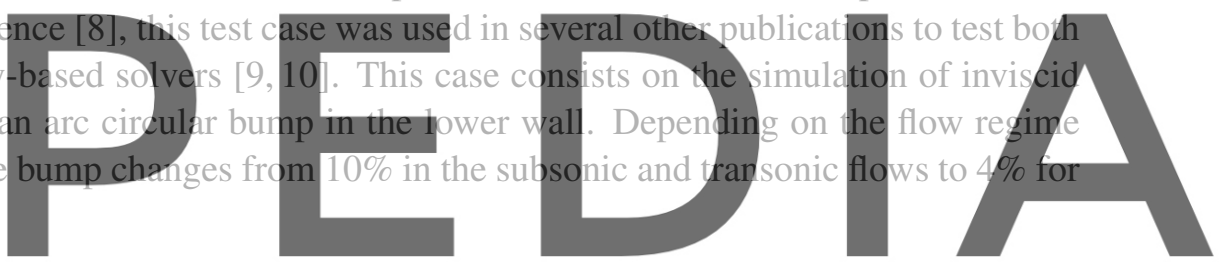

The paper is organized in the following way: Section 2 gives a brief description of the flow solver, the

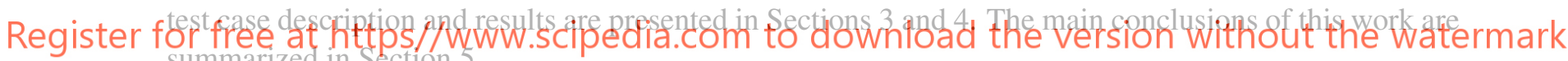 summarized in Section 5.}

\section{ReFRESCO FLOW SOLVER}

The fluid is an inviscid perfect gas, so the vicosity $\mu$, and thermal conductivity coefficient $k_{T}$ are zero, and the pressure $P$ is related to the density $\rho$ and temperature $T$ by the equation of state:

$$
P=\rho R T
$$

where $R=287.025\left[\mathrm{~m}^{2} \mathrm{~s}^{-2} \mathrm{~K}^{-1}\right]$ is the ideal gas constant.

For a fluid with these properties mass conservation, momentum and energy balances are given by the Euler equations:

$$
\begin{aligned}
& \frac{\partial \rho}{\partial t}+\frac{\partial}{\partial x_{j}}\left(\rho u_{i}\right)=0, \\
& \frac{\partial}{\partial t}\left(\rho u_{i}\right)+\frac{\partial}{\partial x_{j}}\left(\rho u_{j} u_{i}\right)=-\frac{\partial P}{\partial x_{i}}, \\
& \frac{\partial E}{\partial t}+\frac{\partial}{\partial x_{j}}\left(\rho u_{j} H\right)=0,
\end{aligned}
$$


where $u_{i}, E$ and $H$ represent the velocity along the $i^{t h}$ direction, total energy and total enthalpy, respectively.

Mass conservation is ensured by means of a pressure-correction equation based on the SIMPLE algorithm. The pressure-velocity-density coupling used in the derivation of the pressure correction equation follows the procedure proposed in [3].

\subsection{Finite volume discretization}

ReFRESCO uses a finite volume method with cell-centered collocated variables in the discretization of transport equations. Grids containing cells with arbitrary geometries, as well as hanging nodes, are possible because of a face-based implementation. Linearization is performed with Picard's method and a segregated approach is adopted to solve the systems of algebraic equations.

Equation (3) presents a general formulation for any transport equation, where $\phi$ represents a scalar quantity, and the values of $\rho, u_{j}$ and $\Gamma$ are assumed to be known:

$$
\frac{\partial}{\partial t}(\rho \phi)+\frac{\partial}{\partial x_{j}}\left(\rho u_{j} \phi\right)=\frac{\partial}{\partial x_{j}}\left(\Gamma \frac{\partial \phi}{\partial x_{j}}\right)+Q
$$

In this equation, it is possible to identify four different terms. The two terms on the left hand side model transient and convection effects, respectively, while the first term in right hand side represents diffusive effects. The term denoted as $Q$ represent sources/sinks terms. Since the selected test case in this paper considers an inviscid fluter

Integrating over a contro

Eq. (3) can be written as:
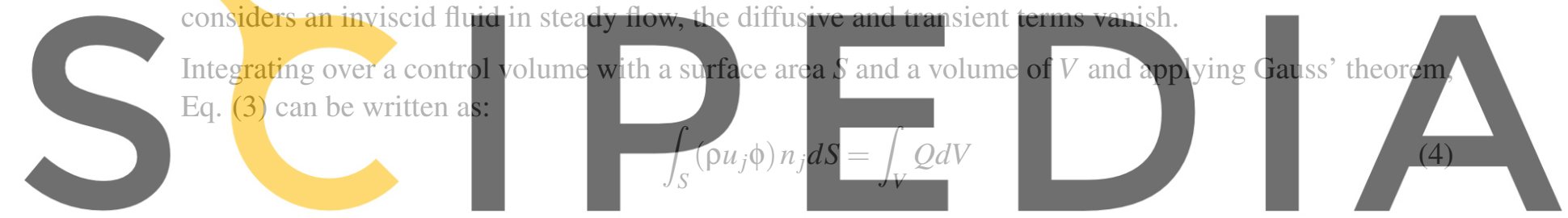

The mid-point rule is used to calculate the surface integral of the cell faces, leading to the discrete form

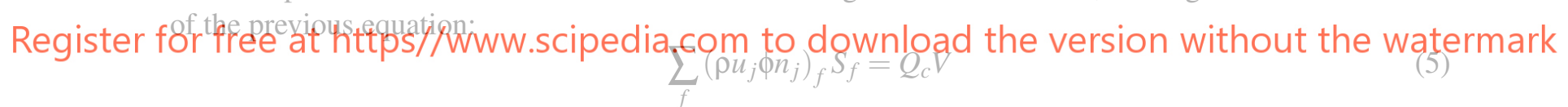

where the summation is over the faces $S_{f}$ of the control volume and the subscript $f$ and $c$ represent the values at face and cell center, respectively, $n_{j}$ represents the outer normal to the cell face.

The interpolation techniques for the value of $\phi$ at faces used in ReFRESCO are described in [11] and are not repeated here

After application of the discretization schemes for all cells in the domain, a system of linear equations is obtained. For a cell $P$ in the interior of the domain, the general form of the linear equation can be written as:

$$
A_{P} \phi_{P}+\sum_{n b} A_{n b} \phi_{n b}=B
$$

where $A_{p}$ and $A_{n b}$ represent the coefficients obtained from the discretization of the equation and $B$ represents all terms included in the right hand side.

The equations above require not only the interpolation of $\phi$, but also of the velocity to the cell faces. It has been proven that on grids with collocated variables the use of linear interpolation results in checkerboard 
oscillations $[12,13]$. To avoid this known issue the velocities used in the calculation of volume or mass fluxes are interpolated to the cell face centers by means of a pressure-weighted interpolation (PWI) [14]:

$$
\left(u_{j}\right)_{f}={\overline{\left(u_{j}\right)_{f}}}-\overline{\left(\frac{V}{A_{P}^{u}}\right)_{f}}\left(\left(\frac{\partial p}{\partial x_{j}}\right)_{f}-\overline{\left(\frac{\partial p}{\partial x_{j}}\right)_{f}}\right)
$$

where the overbar represents a geometrical interpolation and $A_{P}^{u}$ the coefficient of the linearized momentum equation.

\subsection{Compressible SIMPLE algorithm}

As in the incompressible solver, mass conservation is enforced using a SIMPLE like algorithm. In the present solver a non-linear iteration consists of :

1. Assemble and solve the momentum equations for a velocity prediction;

2. Assemble and solve the pressure correction equation for the pressure correction;

3. Correct velocity, density and pressure;

4. Calculate new mass fluxes;

5. Solve the energy equation to obtain the temperature value;

6. Calculate density according to the equation of state Eq. (1).

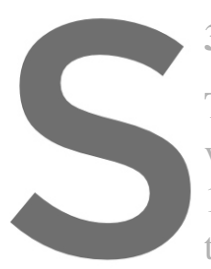

\section{TEST CASE}

The flow throughout a

vergence properties of

1 illustrates the domains
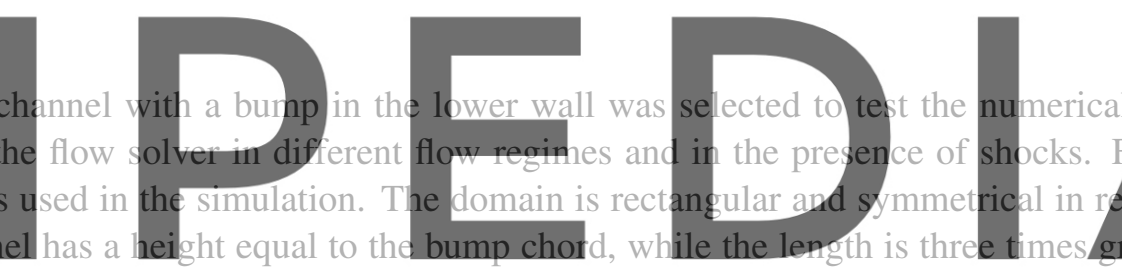

The thickness of the bump depends on the inlet flow conditions, so for the subsonic and transonic flows

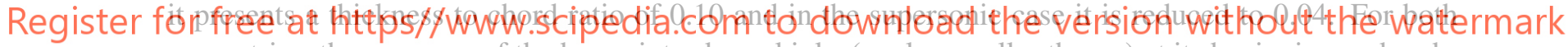
geometries, the presence of the bump introduces kinks (angles smaller than $\pi$ ) at its beginning and end.

Subsonic and transonic flows

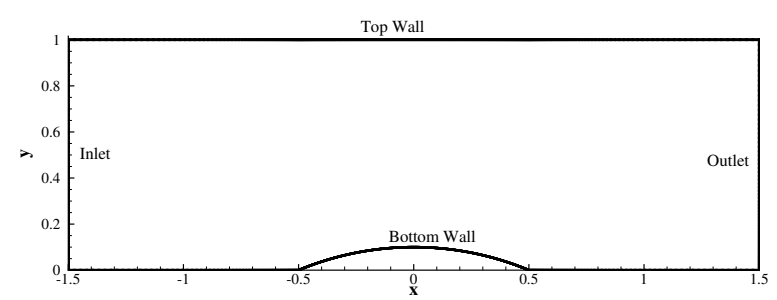

Supersonic flow

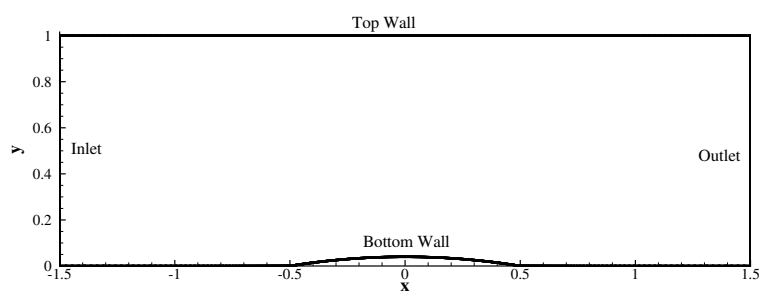

Figure 1: Geometries for the simulation of the flow of an inviscid fluid over a circular-arc (bump).

To obtain a mathematical well posed problem, the Euler equations require the application of consistent boundary conditions in the inlet and outlet boundaries. For the subsonic and transonic flow regimes the flow is subsonic at both these boundaries. So at the inlet the flow is considered uniform and velocity, density and temperature are prescribed, and at the outlet boundary the pressure is specified. The inlet 
velocity is calculated in order to obtain a Mach number of 0.5 for the subsonic flow and 0.675 for the transonic flow. In the case of the supersonic regime the inlet Mach number is 1.65 and the flow is also supersonic at the outlet boundary. Therefore, all dependent variables are specified at the inlet and extrapolated with zero streamwise derivatives at the outlet.

Two sets of geometrically similar grids were generated for the presented domains. Due to the simplicity of the geometry, single-block structured grids have been adopted and so the definition of the grid refinement ratio, $r_{i}$, is trivial:

$$
r_{i}=\sqrt{\frac{\left(N_{\text {cells }}\right)_{1}}{\left(N_{\text {cells }}\right)_{i}}}=\frac{\left(N_{x}\right)_{1}}{\left(N_{x}\right)_{i}}=\frac{\left(N_{y}\right)_{1}}{\left(N_{y}\right)_{i}} .
$$

$N_{\text {cells }}$ is the total number of cells, $N_{x}$ is the number of faces in the horizontal $x$ direction and $N_{y}$ is the number of faces in the vertical $y$ direction.

The grid topology is presented in figure 2 alongside of a detailed view of the grid near the kinks. The grid lines are equally distributed in the vertical direction, but there is clustering of grid lines in the horizontal direction near the bump. For simplicity in the grid generation process, the vertical grid lines are not orthogonal to the bottom boundary. It is also important to point out that all grids have a vertex of the cells at the geometry kinks and so the dependent (cell-centered) variables are not calculated there.

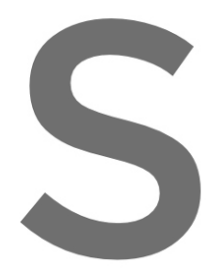

\section{Subsonic and Transonic set $r_{i}=11$}

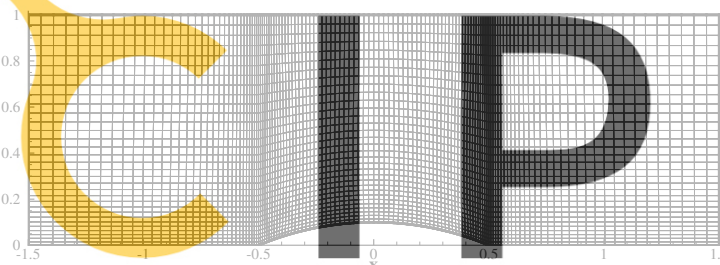

$r_{i}=1$

Register for free at https//www.scipedia.com to

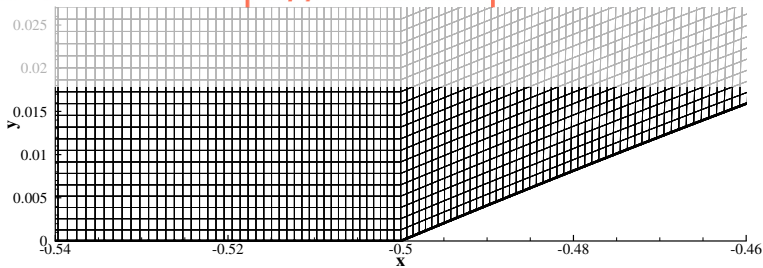

Supersonic set $r_{i}=11$
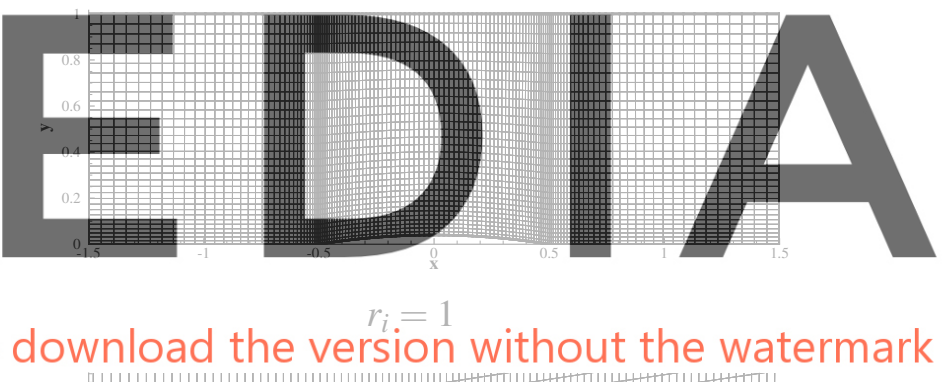

Figure 2: Illustration of the grids for the calculation of subsonic, transonic and supersonic flows of an inviscid fluid over a bump.

Table 1 presents the number of cells $N_{\text {cells }}$, the number of faces on the bump $N_{\text {bump }}$, and the grid refinement ratio, $r_{i}$, for each case. The coarsest grids with $5.5<r_{i}<88$ intend to replicate the typical grid densities found in the publications that address this test case. Since one of the goals of this exercise is to test the robustness of the flow solver, further grid refinement was performed to determine the grid density required to obtain monotonic convergence of the quantities of interest. In the supersonic test case, there were still some doubts about the extrapolations performed with data obtained from grids with $1<r_{i}<4$ 
and so extra grids were generated reaching a refinement level of 0.5 .

\begin{tabular}{|c|c|c|c|c|c|c|}
\hline \multirow{2}{*}{ Grid } & \multicolumn{2}{|c|}{ Subsonic and Transonic } & \multicolumn{3}{c|}{ Supersonic } \\
\cline { 2 - 7 } & $N_{\text {cells }}$ & $N_{\text {bump }}$ & $r_{i}$ & $N_{\text {cells }}$ & $N_{\text {bump }}$ & $r_{i}$ \\
\hline $2816 \times 704$ & - & - & - & $1,982,464$ & 1,408 & 0.5 \\
$2184 \times 546$ & - & - & - & $1,192,464$ & 1,092 & 0.645 \\
$1636 \times 409$ & - & - & - & 669,124 & 818 & 0.861 \\
$1408 \times 352$ & 495,616 & 704 & 1.0 & 495,616 & 704 & 1.0 \\
$1024 \times 256$ & 262,144 & 512 & 1.375 & 262,144 & 512 & 1.375 \\
$704 \times 176$ & 123.904 & 352 & 2.0 & 123,904 & 352 & 2.0 \\
$512 \times 128$ & 65,536 & 256 & 2.75 & 65,536 & 256 & 2.75 \\
$352 \times 88$ & 30,976 & 176 & 4.0 & 30,976 & 176 & 4.0 \\
$256 \times 64$ & 16,384 & 128 & 5.5 & 16,384 & 128 & 5.5 \\
$128 \times 32$ & 4,096 & 64 & 11 & 4,096 & 64 & 11 \\
$64 \times 16$ & 1,024 & 32 & 22 & 1,024 & 32 & 22 \\
$32 \times 8$ & 256 & 16 & 44 & 256 & 16 & 44 \\
$16 \times 4$ & 64 & 8 & 88 & 64 & 8 & 88 \\
\hline
\end{tabular}

Table 1: Total number of cells $N_{\text {cells }}$, number of boundary faces in the bump $N_{\text {bump }}$ and grid refinement ratio $r_{i}$ of the grids for subsonic, transonic and supersonic flow simulations.

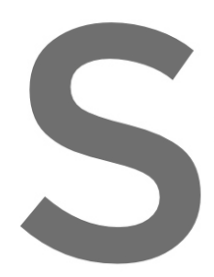

To fully describe the te

the simulation: the initail

For the coarsest grids, the

variables are obtained wit
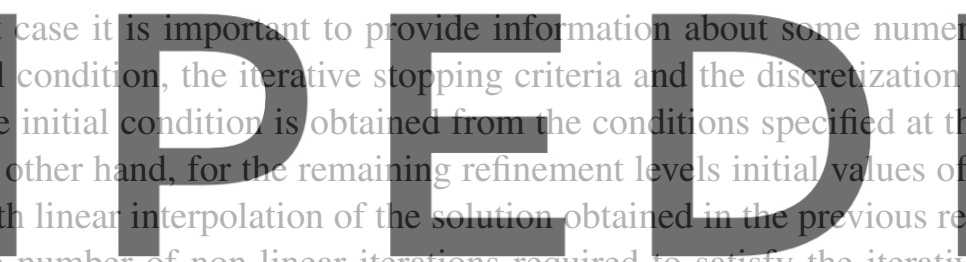

This strategy reduces the number of

f non-linear iterar

criteria, but it is not essential to achieve iterative convergence. In the present study, it is considered that

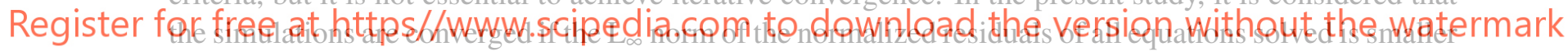

than $10^{-8}$. The residuals are normalized with the main diagonal of the systems of linear equations $A_{P}$

multiplied by reference quantities related to each equation. Quantities specified at the inlet are used for reference quantities. This means that the change in normalized residuals correspond to the change in dimensionless variables in a simple Jacobi iteration. The discretization schemes of the convective term of the transport equations are: the Fromm scheme with an harmonic limiter (HARM); limited QUICK scheme; central-differencing scheme blended with 10\% of first-order upwind (CDS-10UP); and firstorder upwind (UP). The HARM and QUICK schemes are a standard choice for incompressible flow simulations, whereas the CDS-10UP and UP schemes have been used in similar studies published in the literature $[4,5]$.

\section{RESULTS}

To analyze the performance of the flow solver is important to distinguish between numerical robustness, i.e., iterative convergence, and solution accuracy, meaning grid convergence properties. The first was studied based on the evolution of the $\mathrm{L}_{\infty}$ norm of the normalized residuals, while several quantities of interest were studied to analyze the grid convergence properties of the solver. The quantities of interest 
shown in the present work are:

- Mach number, $M a=\|u\| / a$ with speed of sound $a=\sqrt{\gamma p / \rho}$, field and distributions along the lower wall;

- Force coefficient $C_{x}$ on the bottom wall defined by:

$$
C_{x}=\frac{\int_{-1.5}^{1.5} p d y}{1 / 2 \rho_{r e f} V_{r e f}^{2} L_{r e f}},
$$

where reference flow quantities are obtained from the inlet conditions and $L_{r e f}=3 \mathrm{~m}$.

The Mach number is a standard choice of this type of studies because it is the dimensionless quantity that identifies the flow regime. The force coefficient along the $x$ direction, $C_{x}$ is a functional quantity that illustrates the global convergence properties of the solution. For the subsonic flow, as the flow is symmetric, its value is known and equal to zero.

Before considering the grid convergence properties of the solver, it is important to ensure sufficient iterative convergence of the numerical simulations. Figure 3 shows the typical evolution of the residuals on a fine $\left(r_{i}=2\right)$ and medium $\left(r_{i}=5.5\right)$ grid. The simulations performed on the other grid levels and with other discretization schemes present similar iterative convergence. So, it can be concluded that the simulations can be converged to the desired level for all considered grid densities, discretization schemes, and most importantly flow regimes.
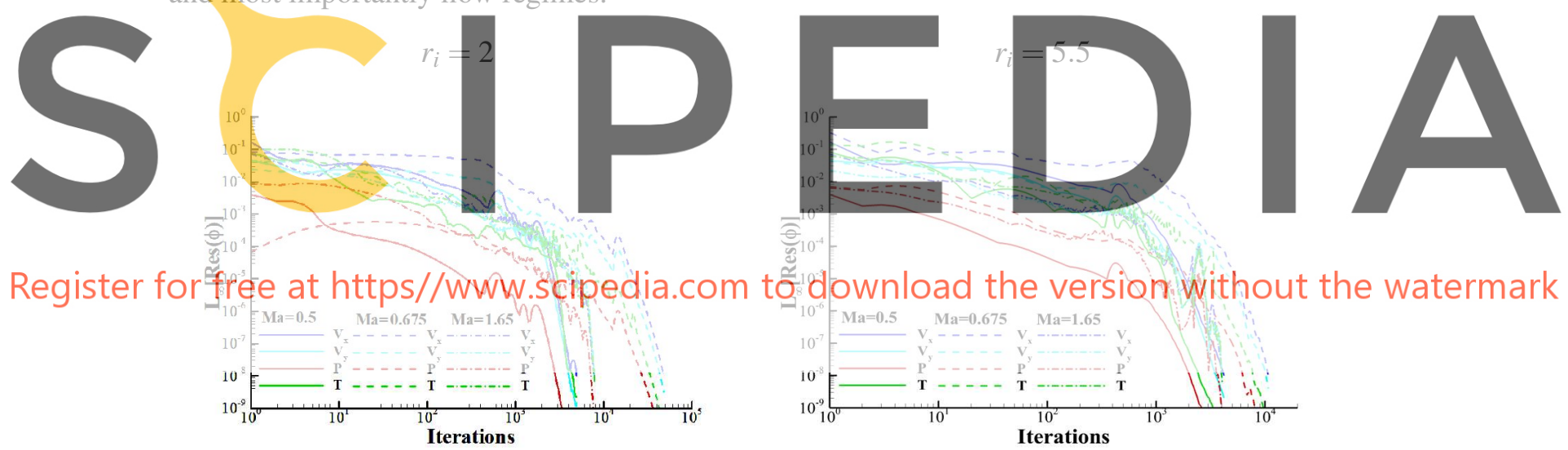

Figure 3: Iterative convergence of ReFRESCO for the calculation of subsonic, transonic and supersonic flows for two different grid refinement levels using the HARM scheme in the discretization of the convective terms.

\subsection{Mach Number}

Figures 4, 5 and 6 present the isolines of the Mach number obtained in the finest grid (solid lines) and a coarse grid with $r_{i}=11$ (dashed lines) using the four convection schemes tested. The distribution of the Mach number on the bottom wall is illustrated in figure 7.

For the subsonic, figure 4, and transonic, figure 5, flow regimes the influence of the grid refinement 


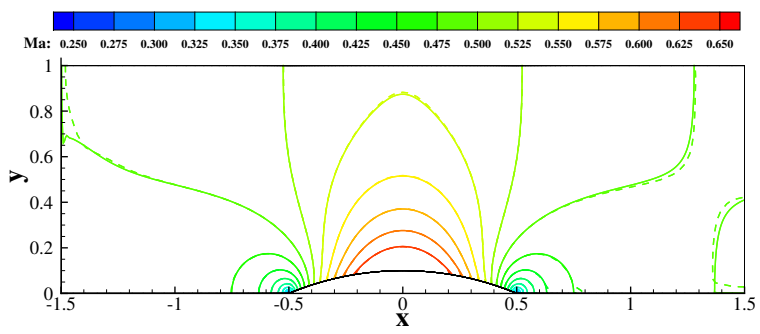

(a) HARM

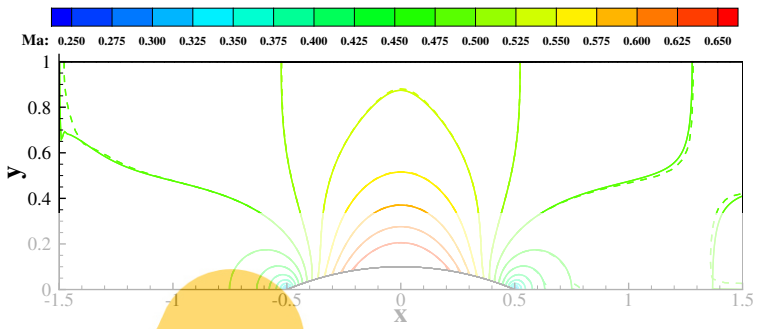

(c) QUICK

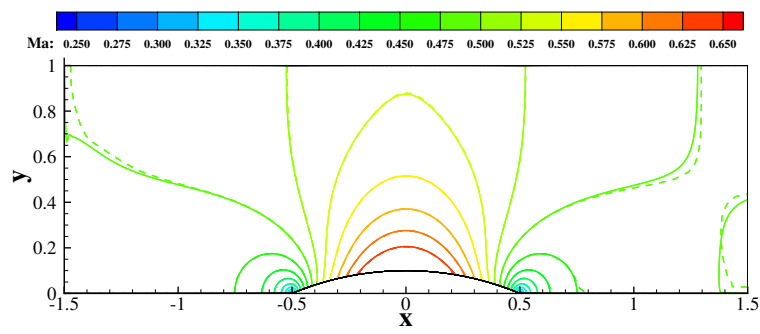

(b) CDS-10UP

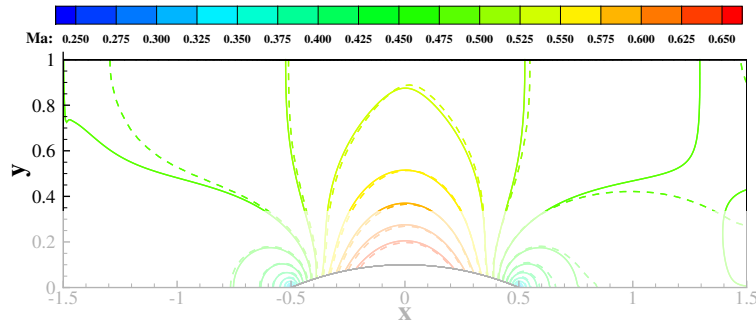

(d) UP

Figure 4: Mach distributions using different convection schemes for the subsonic flow of an inviscid fluid over a bump. Solid lines $r_{i}=1$; Dashed lines $r_{i}=11$.
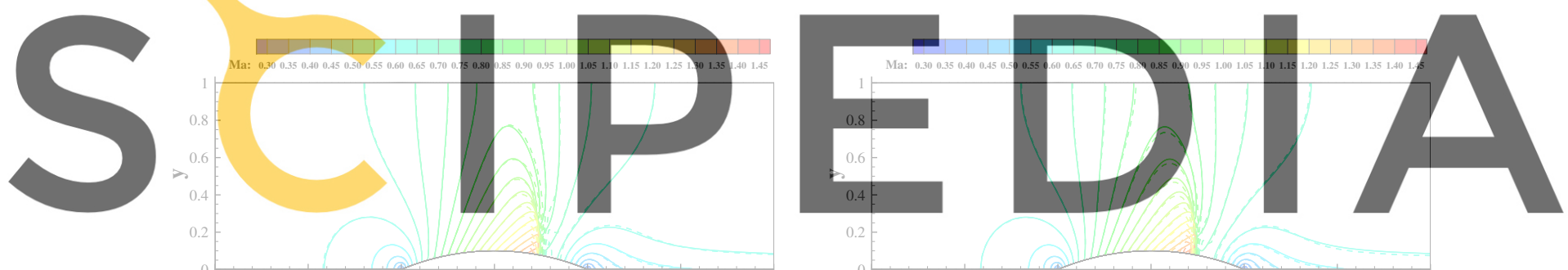

Register for free at https//wn'w.scipedia.com to download the version without the watermark

(a) HARM

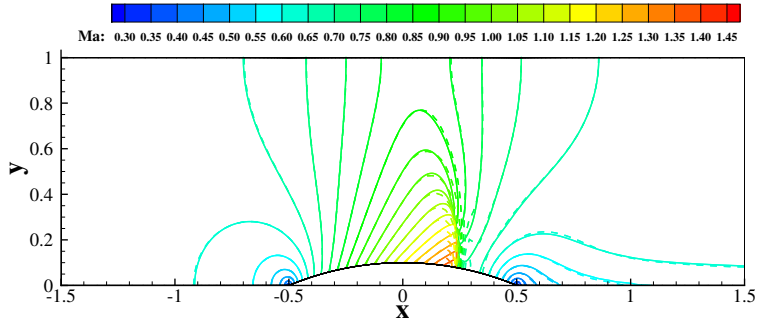

(c) QUICK (b) CDS-10UP

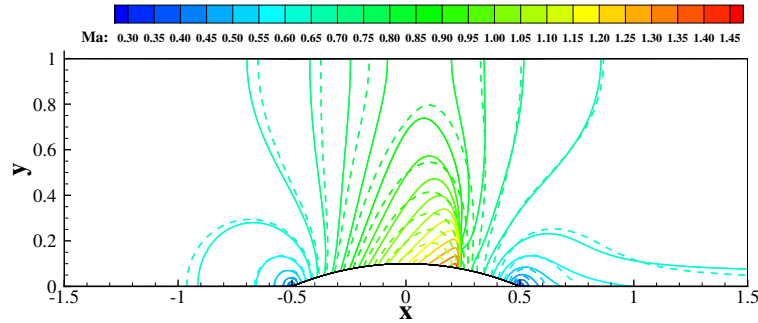

(d) UP

Figure 5: Mach distributions using different convection schemes for the transonic flow of an inviscid fluid over a bump. Solid lines $r_{i}=1$; Dashed lines $r_{i}=11$.

seems small, but there are a few details that are affected by grid density. In the subsonic case the main 


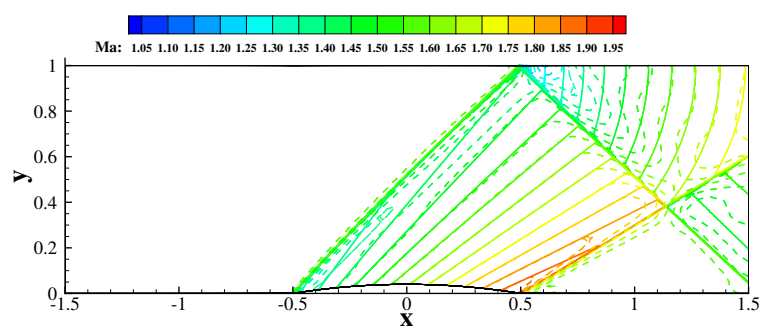

(a) HARM

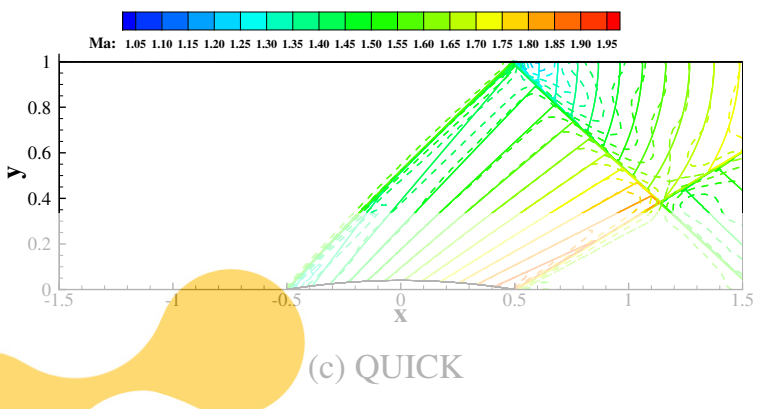

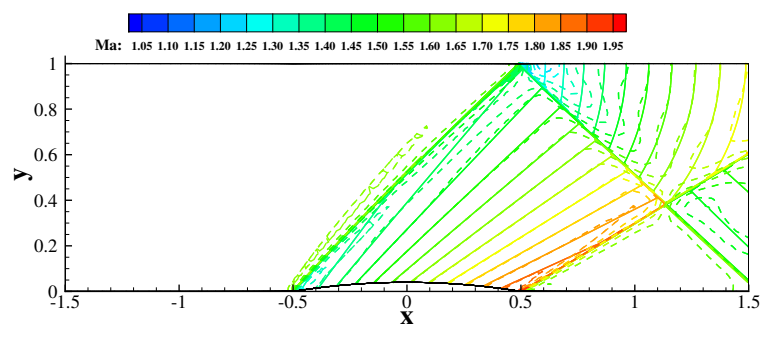

(b) CDS-10UP

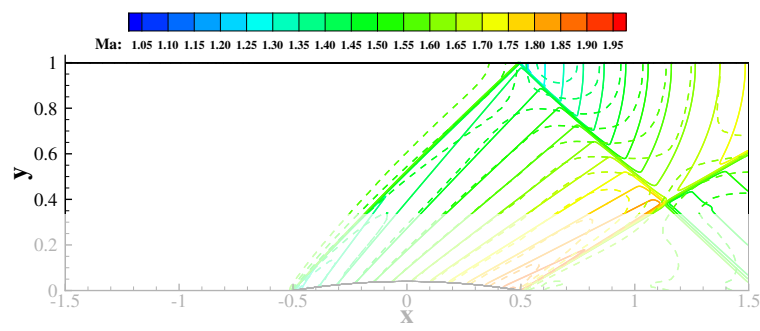

(d) UP

Figure 6: Mach distributions using different convection schemes for the transonic flow of an inviscid fluid over a bump. Solid lines $r_{i}=0.5$; Dashed lines $r_{i}=11$.
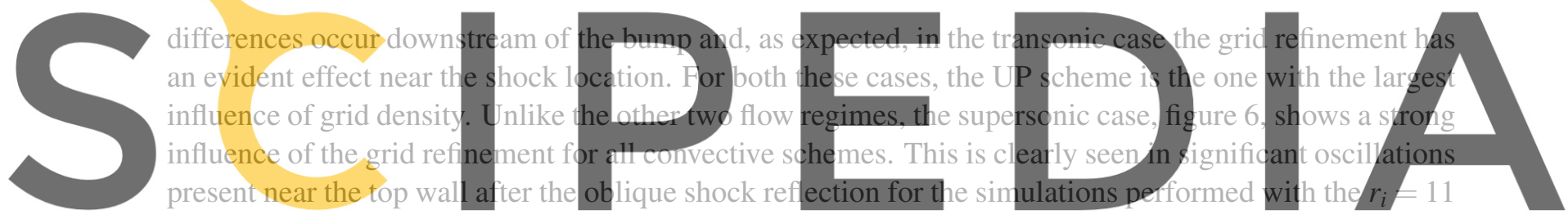
grid. The Mach number distribution in the lower wall presented in figure 7 confirm the trends observed

Register for theree at ipaltpes//www.scipedia.com to download the version without the watermark

\subsection{Grid convergence of $C_{x}$}

The observed order of grid convergence $p$ of the horizontal force coefficient $C_{x}$ is determined using the power series expansions proposed in [15]. The least-squares fits are performed with the data of the four finest grids, which means $1 \leq r_{i} \leq 2.75$ for the subsonic and transonic flows and $0.5 \leq r_{i} \leq 1$ for the supersonic flow. Figure 8 illustrates the convergence of the force coefficient with grid refinement.

For subsonic flow, figure 8a, the observed order of grid convergence, $p$, is between $p=1$ (UP and CDS+0.1UP) and $p=1.6$ (HARM and QUICK), which is in agreement with the expected value. The estimate of the exact solution is consistent for the four schemes tested and it satisfies the exact value $C_{x}=0$. The observed orders of accuracy obtained for the transonic flow, figure $8 \mathrm{~b}$, show awkward result for the UP scheme with $p=1.6$. However, the data suggest that the value of $p$ will change if finer grids are used with the UP scheme. On the other hand the four estimates of the exact solution (values for $r_{i}=0$ ) are very similar. For the supersonic flow, figure 8c, the convergence of $C_{x}$ with $r_{i}$ only becomes monotonic for $r_{i}<3$ and so the three extra grids with $r_{i}<1$ are essential to obtain reliable estimates of the exact solution. The oblique shocks at the kinks of the bump, lead to a significant reduction of 


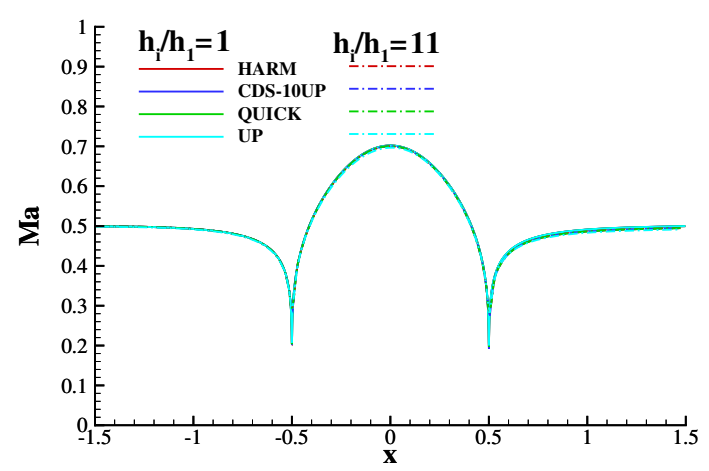

(a) Subsonic

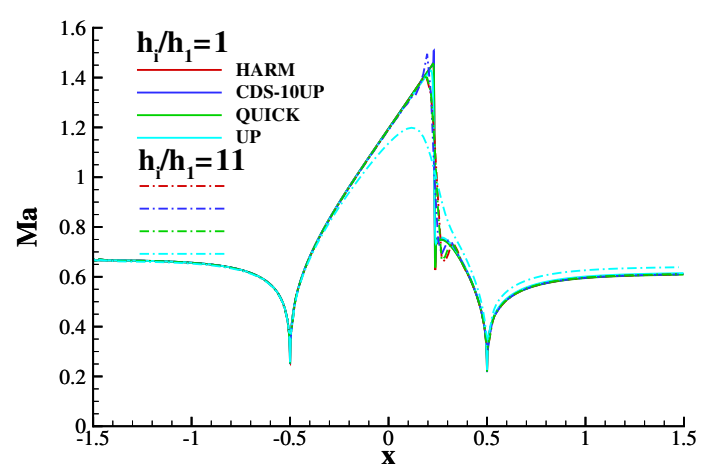

(b) Transonic

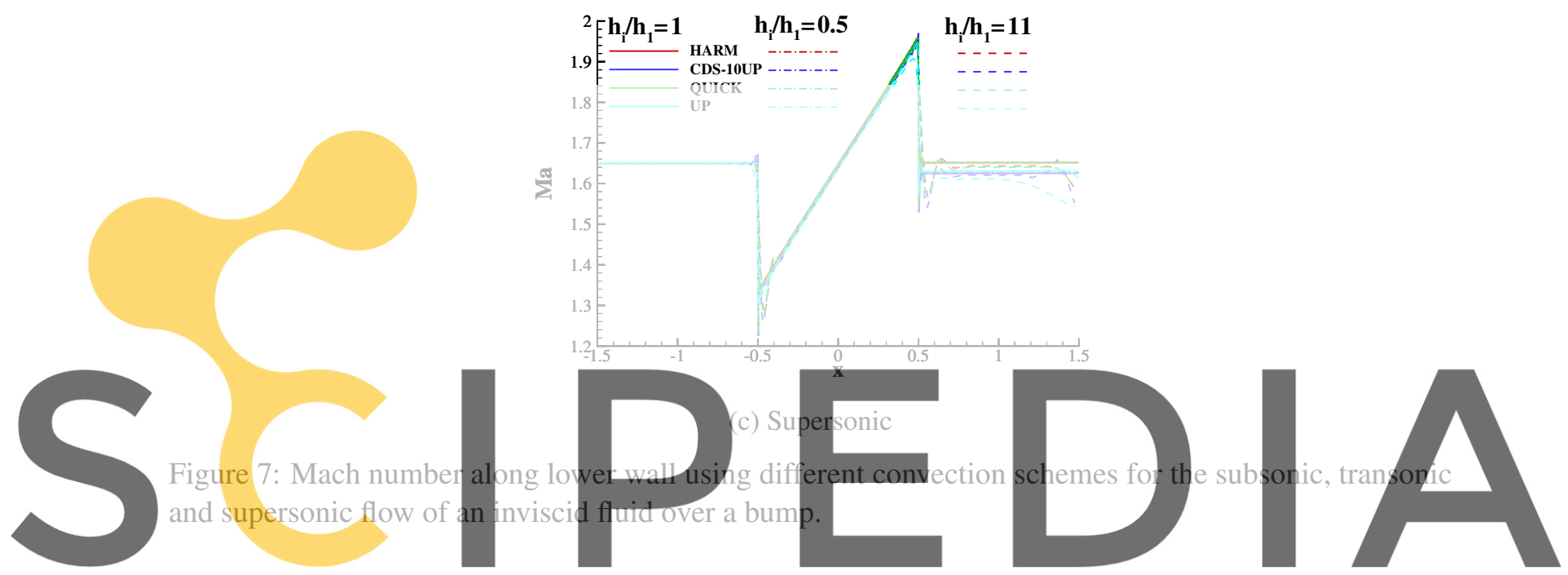

the values of $p$ obtained for the HARM and QUICK schemes with observed values clearly below 1.

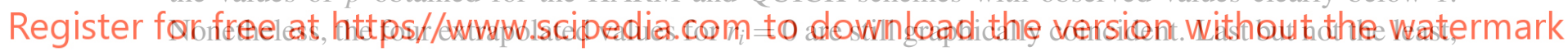

the results show a significant increase of the error level for the UP scheme when compared with the other

schemes. Solutions for the finest grids obtained with the UP scheme exhibit larger numerical errors than those obtained with the HARM and QUICK schemes in grids up to $r_{i}=5.5$.

\section{CONCLUSIONS}

This paper presents a verification study for the standard GAMM test case consisting of the flow of an inviscid ideal gas over a bump in a channel at different Mach numbers. The objective of this study is twofold: check the robustness of the newly developed compressible flow solver (ability to reduce iterative errors to negligible levels) and assess the grid convergence properties of several quantities of interest. Therefore, simulations with four convection discretization schemes in geometrically similar grids covering a wide range of grid densities were performed for three different flow regimes: subsonic, transonic and supersonic.

The main conclusions drawn from the results are:

- Although the iterative convergence slows down at higher Mach numbers and on finer grids, all 


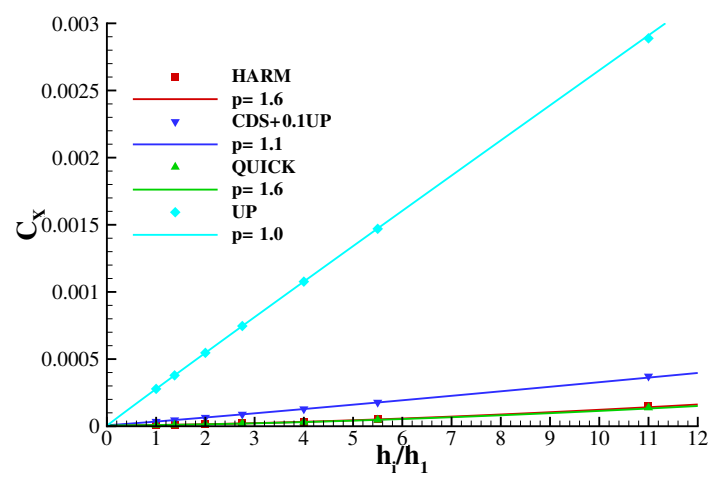

(a) Subsonic

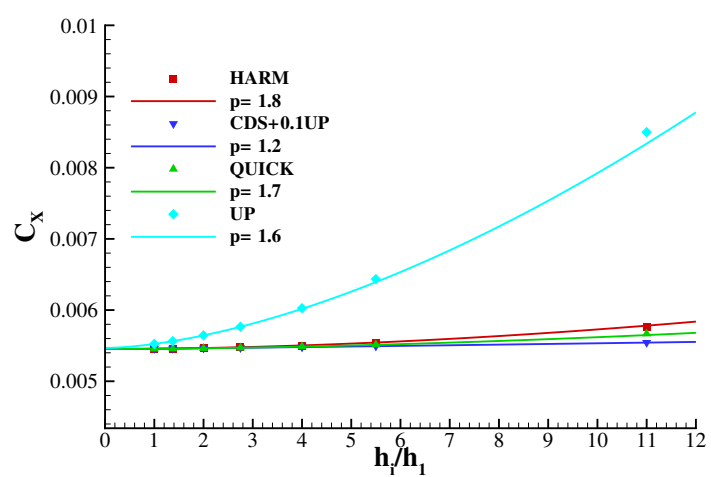

(b) Transonic

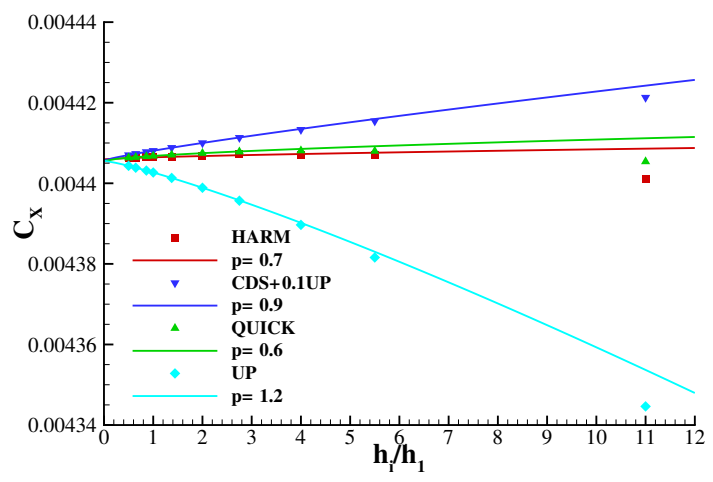

(c) Supersonic

Figure 8: Grid convergence of the horizontal $C_{x}$ force coefficient obtained by integration of the pressure on the bottom wall. ReFRESCO results using different convection schemes for the subsonic, transonic and supersonic flows of an inviscid fluid over a bump.

simulations could be iteratively converged to residual levels close to machine accuracy, which demonstrates the robustness of the pressure-based solver;

- Orders of grid convergence between one and two were observed depending on the flow regime, discretization scheme and quantity of interest;

- Significantly more refined grids that those found in the open literature were required to obtain monotonic convergence;

- Lowest grid convergence rates were obtained for the supersonic flow.

Last but not the least, oscillations are obtained downstream of the shocks with the four schemes tested. However, the present flow solver does not use gradient limiters or other specialized techniques for high Mach number flows in view of the target combination with an incompressible multi-phase solver for maritime applications. 


\section{ACKNOWLEDGEMENTS}

The authors wish to acknowledge the FCT - Fundação para a Ciência e a Tecnologia, through grant with reference SFRH/BD/137326/2018 granted to the first author, and the Dutch Ministry of Economic Affairs for their support in the present research.

\section{REFERENCES}

[1] Eça, L., Klaij, C.M., Vaz, G., Hoekstra, M., and Pereira, F.S. On code verification of RANS solvers. Journal of Computational Physics (2016) 310:418 - 439.

[2] Roache, P.J. Verification and Validation in Computational Science and Engineering. Hermosa Publishers, Albuquerque, NW (1998).

[3] Muralha, J., Eça, L., and Klaij, C.M. Code Verification of a Pressure-Based Solver for Subsonic Compressible Flows. Journal of Verification, Validation and Uncertainty Quantification (2020) $5(4)$.

[4] Demirdžić, I., Lilek, Ž., and Perić, M. A Collocated Finite Volume Method For Predicting Flows at All Speed. Journal of Numerical Methods in Fluids (1993) 16(12):1029-1050.

[5] Moukalled, F. and Darwish, M. A High-Resolution Pressure-Based Algorithm for Fluid Flow at All Speeds. Journal of Computational Physics (2001) 168(1):101 - 130.

[6] Choi, D. and Merkle, C.L. Application of time-iterative schemes to incompressible flow. AIAA Journal (1985) 23(10):1518-1524.

[7] Edwards, J.R. and Liou, M.S. Low-diffusion flux-splitting methods for flows at all speeds. AIAA journal (1998) 36(9):1610-1617.

[8] Rizzi, A. and Viviand, H., editors. Numerical Methods for the Computation of Inviscid Transonic Flows with Shock Waves. Number 3 in Notes on Numerical Fluid Mechanics. Vieweg (1981).

[9] Ni, R.H. A multiple-grid scheme for solving the euler equations. AIAA Journal (1982) 20(11):1565-1571.

[10] Favini, B., Broglia, R., and Di Mascio, A. Multigrid acceleration of second-order eno schemes from low subsonic to high supersonic flows. International Journal for Numerical Methods in Fluids (1996) 23(6):589-606.

[11] Waterson, Nicholas P. and Deconinck, Herman. Design Principles for Bounded Higher-Order Convection Schemes-a Unified Approach. Journal of Computational Physics (2007) 224(1):182-207.

[12] Ferziger, J.H. and Perić, M. Computational Methods for Fluid Dynamics. Springer-Verlag, 3rd edition (2001).

[13] Wesseling, P. Principles of Computational Fluid Dynamics. Springer, Berlin, Germany (2001).

[14] Rhie, C.M. and Chow, W.L. Numerical study of the turbulent flow past an airfoil with trailing edge separation. AIAA Journal (1983) 21(11):1525-1532.

[15] Eça, L. and Hoekstra, M. A procedure for the estimation of the numerical uncertainty of cfd calculations based on grid refinement studies. Journal of Computational Physics (2014) 262:104 130. 